\title{
The Herrenhausen Symposium on Neurodegeneration
}

\begin{abstract}
Although the pathological hallmarks of neurodegenerative diseases have been described for over 100 years, our understanding of the molecular events leading to neuronal death has emerged over the past two decades. Despite all of this progress in basic research, why do we still lack disease-modifying therapeutics?
\end{abstract}

$\mathbf{T}$ rying to identify obstacles to translational research in neurodegeneration, Nature Medicine, in conjunction with the Volkswagen Foundation, organized the inaugural Herrenhausen Symposium on Neurodegeneration. On the basis of consultations with over 140 experts, we invited opinion leaders in the field to a round-table discussion in Seeon, Germany. The commentaries in this collection summarize the key points from the scientific sessions at the meeting and seek not only to identify translational roadblocks, but also to provide suggestions on how to advance the field.

Chief among the problems facing researchers today is insufficient knowledge of the mechanisms of disease. Christian Haass examines the proximal causes of neurodegeneration-initiation and propagation of disease-and describes how examining mechanisms of disease shared by the various neuropathological conditions could lead to new therapeutic strategies.

The other side of degeneration is repair. In his contribution, Eng Lo posits that, in the face of extensive neurodegeneration, endogenous mechanisms of compensation, repair and remodeling may occur. Research into cross-talk between neurons, glia and the vasculature in the context of known risk factors (such as aging and vascular disease) may unravel new mechanisms of degeneration and repair and identify optimal time windows for treatment.

The identification of mutations in genes leading to autosomal-dominant forms of disease has helped us understand pathogenic cascades in several neurodegenerative diseases and spurred development of transgenic animal models of these diseases. Following a wave of genome-wide association studies and advances in next-generation sequencing methods, Christine Van Broeckhoven comments on how to interpret these recent findings, what we can learn from continually examining early-onset forms of disease and, moving forward, how carefully designed genetic studies can increase the chance of identifying robust genetic associations.
The development of transgenic models of disease has undoubtedly contributed greatly to our understanding of disease. Mathias Jucker comments on the benefits of these models and argues that, despite their limitations, incomplete models of disease pathogenesis can be used to examine specific disease processes. However, success in using these models for preclinical testing will depend on asking the right questions of the models and acknowledging their limitations.

Several promising disease-modifying agents have failed to show effects on primary outcome measures in clinical trials. Anthony Lang discusses several obstacles in planning and conducting clinical trials, including enrollment of patients with fairly advanced disease, and, in terms of the therapeutic effects, whether heterogeneity at the clinical, genetic and pathological levels contributes to variable responses to therapy. One way to improve these trials, argues Kaj Blennow, is to incorporate biomarkers to diagnose patients early and stratify patient cohorts on the basis of their response to therapy. 'Theragnostic' biomarkers should also be developed for future trials to ascertain whether drugs are acting on their relevant target.

Future success in translational research on neurodegenerative disease is beset with problems in bridging the gap between academia and the pharmaceutical industry. In his commentary, Steven Finkbeiner suggests collaboration needs to be fostered between academia and industry, as well as between academics across disciplines. By providing academics with resources and incentives to conduct translational research, promising leads can be developed at a lower risk to investors, fostering drug development in an area that desperately needs new medicines.

These commentaries are intended as a vehicle to stimulate further discussion among scientists from all areas of neurodegenerative disease research, encouraging cross-talk across disciplines and among diseases. We hope these articles make the case that there is hope that, in the near future, discoveries made at the bench will translate into new drugs at the bedside. 